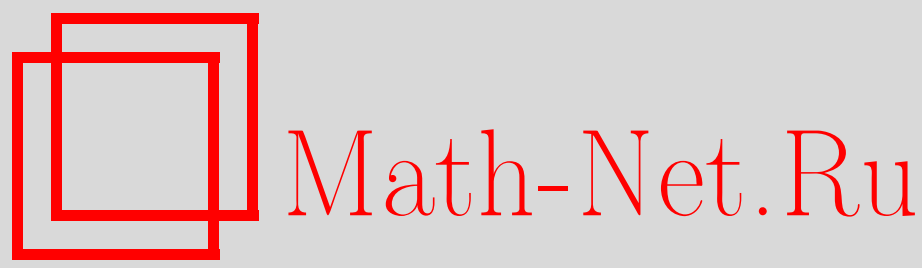

П. А. Глушак, Б. Б. Маркив, М. В. Токарчук, Метод неравновесного статистического оператора Зубарева в обобщенной статистике многочастичных систем, $Т M \Phi$, 2018, том 194, номер 1, 71-89

DOI: https://doi.org/10.4213/tmf9389

Использование Общероссийского математического портала Math-Net.Ru подразумевает, что вы прочитали и согласны с пользовательским соглашением http://www . mathnet.ru/rus/agreement

Параметры загрузки:

IP : 3.85 .5 .30

26 апреля 2023 г., $14: 28: 10$

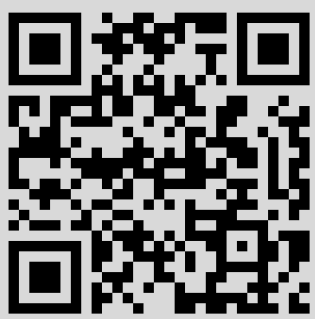




\title{
МЕТОД НЕРАВНОВЕСНОГО СТАТИСТИЧЕСКОГО ОПЕРАТОРА ЗУБАРЕВА В ОБОБЩЕННОЙ СТАТИСТИКЕ МНОГОЧАСТИЧНЫХ СИСТЕМ
}

\begin{abstract}
Представлено обобщение метода неравновесного статистического оператора Зубарева на основании принципа максимума энтропии Реньи. В рамках этого подхода получены уравнения переноса для основного набора параметров сокращенного описания неравновесных процессов в классической системе взаимодействующих частиц, при этом использовались уравнения Лиувилля в дробных производных. Для классических систем частиц в среде с фрактальной структурой получено немарковское уравнение диффузии с пространственными дробными производными. В случае конкретного моделирования частотной зависимости функции памяти получено обобщенное уравнение диффузии типа Кеттано с учетом пространственной и временной фрактальности. Представлено обобщение неравновесной термополевой динамики в методе неравновесного статистического оператора Зубарева в рамках статистики Реньи.
\end{abstract}

Ключевые слова: энтропия Реньи, неравновесный статистический оператор, обобщенные уравнения переноса, уравнения диффузии.

DOI: https://doi.org/10.4213/tmf9389

\section{1. ВВЕДЕНИЕ}

Метод коллективных переменных [1], метод временны́х функций Грина [2] и метод неравновесного статистического оператора (HCO) [3]-[9], предложенные Д. Н. Зубаревым, имеют огромное значение в статистической физике систем взаимодействующих частиц. Метод коллективных переменных получил свое развитие в работах И. Р. Юхновского в равновесной теории электролитов [10]-[13], в теории фазовых переходов [14]-[16], квантовых ферми- и бозе-систем [13], [17]-[19]. Метод временнь́х функций Грина получил широчайшее применение в статистической теории конденсированных систем (см., например, [20]-[32]). Изящный метод НСО является наиболее общим в современной неравновесной статистической механике, поскольку из него

* Институт физики конденсированных систем НАН Украины, Львов, Украина. E-mail: mtoc2010@ukr.net, mtoc@icmp.lviv.ua

${ }^{\dagger}$ GlobalLogic Украина, Львов, Украина 
можно получить другие важные подходы, основанные на применении проекционных операторов Мори и Кавасаки-Гантона [5], [33]. Он использовался в теории твердого тела при описании диэлектрических и магнитных свойств, процессов переноса в полупроводниках [4], [8], [9], [29], [32]-[35], в теории жидкого состояния и плотных газов при построении обобщенной гидродинамики для простых жидкостей [36]-[40], полярных и магнитных жидкостей [41]-[44], ионных жидкостей [45]-[47], плотной плазмы [48], [49] и полуквантового гелия [50], [51]. Метод НСО применяется в теории каталитических процессов на поверхности металлов [52], при построении релятивистской гидродинамики и термодинамики [53]-[58], а также в неравновесной термополевой динамике [59], [60].

Важно отметить две работы Зубарева и Климова [61], [62] по физике плазмы и управляемого термоядерного синтеза, которые фактически положили начало развитию целого направления физики пристеночной плазмы [63], [64]. Это направление актуально и сегодня в связи с проблемой пылевой плазмы в ТОКАМАКах и других установках плазмы [65]-[68].

В работах Зубарева, Морозова, Омеляна, Токарчука развита концепция согласованного описания кинетических и гидродинамических процессов в плотных газах, жидкостях и плазме [49], [69]-[73], предложенная в работе [74]. Эта концепция получила дальнейшее обобщение с учетом нелинейных гидродинамических флуктуаций [75]-[77], важных в теории турбулентности и динамике фазовых переходов. В исследованиях [76]-[78] метод НСО и метод коллективных переменных применялись совместно.

В работах [79]-[81] метод НСО получил обобщение в статистике Реньи [82]-[84]. Важно отметить работы Луцци и др. [85], [86], где метод НСО и энтропия Реньи используются для описания систем, далеких от равновесия.

Ниже мы кратко изложим метод $\mathrm{HCO}$ в статистике Реньи, а также затронем две задачи, которые обсуждались одним из авторов (М. В. Токарчук) с Д. Н. Зубаревым в феврале и мае 1992 года. Первая задача связана с уравнением Лиувилля в дробных производных, предложенным Нигматулиным [87]. Вторая задача - это формулировка и применение неравновесной термополевой динамики в методе НСО [59].

В разделе 2 сформулирован метод НСО Зубарева в статистике Реньи. В разделе 3 на основании представленного подхода получены уравнения переноса в пространственно-временны́х дробных производных для классической системы частиц с использованием уравнений Лиувилля в дробных производных. В разделе 4 изложена неравновесная термополевая динамика метода НСО в статистике Реньи.

\section{2. МЕТОД НСО ЗУБАРЕВА В СТАТИСТИКЕ РЕНЬИ}

В современных исследованиях нелинейных кинетических и гидродинамических процессов открытых систем [83] используются подходы, основанные на статистике Цаллиса [88], [89], Реньи [82], [90], Шармы-Миттала [91], [92], а также на суперстатистике [93], [94]. Особый интерес составляет обобщение метода НСО в случае статистики Реньи [79].

В методе НСО Зубарева, когда параметры сокращенного описания $\left\langle\widehat{P}_{n}\right\rangle^{t}$ выбраны согласно идее Боголюбова, НСО системы $\varrho\left(x^{N} ; t\right)=\varrho\left(x_{1}, \ldots, x_{N} ; t\right)$ может 
быть найден в общем виде с учетом проецирования как решение уравнения Лиувилля [5], [8], [9]:

$$
\varrho\left(x^{N} ; t\right)=\varrho_{\text {rel }}\left(x^{N} ; t\right)-\int_{-\infty}^{t} e^{\varepsilon\left(t^{\prime}-t\right)} T\left(t, t^{\prime}\right)\left[1-P_{\text {rel }}\left(t^{\prime}\right)\right] i L_{N} \varrho_{\text {rel }}\left(x^{N} ; t^{\prime}\right) d t^{\prime},
$$

где

$$
T\left(t, t^{\prime}\right)=\exp _{+}\left(-\int_{t^{\prime}}^{t}\left[1-P_{\text {rel }}\left(t^{\prime}\right)\right] i L_{N} d t^{\prime}\right)
$$

- оператор эволюции с учетом проецирования, $\exp _{+}-$упорядоченная экспонента, $\varepsilon \rightarrow+0$ после предельного термодинамического перехода, $i L_{N}-$ оператор Лиувилля системы взаимодействующих частиц. Здесь также использованы следующие обозначения: $N$ - полное число частиц системы, $x_{j}=\left\{\vec{p}_{j}, \vec{r}_{j}\right\}$ - фазовые переменные $j$-й частицы, $P_{\text {rel }}\left(t^{\prime}\right)$ - обобщенный проекционный оператор Кавасаки-Гантона, структура которого зависит от формы релевантного статистического оператора:

$$
P_{\text {rel }} \varrho^{\prime}=\left(\varrho_{\text {rel }}(t)-\sum_{n} \frac{\delta \varrho_{\text {rel }}(t)}{\delta\left\langle\widehat{P}_{n}\right\rangle^{t}}\left\langle\widehat{P}_{n}\right\rangle^{t}\right) \int d \Gamma_{N} \varrho^{\prime}+\sum_{n} \frac{\delta \varrho_{\text {rel }}(t)}{\delta\left\langle\widehat{P}_{n}\right\rangle^{t}} \int d \Gamma_{N} \widehat{P}_{n} \varrho^{\prime},
$$

$\varrho_{\text {rel }}\left(x^{N} ; t\right)$ - релевантный статистический оператор, который при $t \rightarrow-\infty$ равен $\varrho\left(x^{N} ; t\right)$. Используя метод Лагранжа, будем искать $\varrho_{\mathrm{rel}}\left(x^{N} ; t\right)$ из условия максимума функционала энтропии Реньи [79]

$$
S_{\mathrm{R}}(\varrho)=\frac{1}{1-q} \ln \int d \Gamma_{N} \varrho^{q}(t)
$$

при фиксированных параметрах сокращенного описания и с учетом условия нормировки. Соответствующий функционал имеет следующий вид:

$$
L_{\mathrm{R}}(\varrho)=\frac{1}{1-q} \ln \int d \Gamma_{N} \varrho^{q}(t)-\alpha \int d \Gamma_{N} \varrho(t)-\sum_{n} F_{n}(t) \int d \Gamma_{N} \widehat{P}_{n} \varrho(t),
$$

где $F_{n}(t)$ - множители Лагранжа. Приравнивая к нулю функциональную производную $\delta L_{\mathrm{R}}(\varrho) / \delta \varrho=0$ и определяя параметр

$$
\alpha=\frac{q}{1-q}-\sum_{n} F_{n}(t)\left\langle\widehat{P}_{n}\right\rangle^{t},
$$

получим релевантный статистический оператор в следующем виде [79]:

$$
\varrho_{\text {rel }}(t)=\frac{1}{Z_{\mathrm{R}}^{*}}\left(1-\frac{q-1}{q} \sum_{n} F_{n}^{*}(t) \widehat{P}_{n}\right)^{1 /(q-1)}
$$

со статистической суммой

$$
Z_{\mathrm{R}}^{*}(t)=\int d \Gamma_{N}\left(1-\frac{q-1}{q} \sum_{n} F_{n}^{*}(t) \widehat{P}_{n}\right)^{1 /(q-1)}
$$

и перенормированными множителями

$$
F_{n}^{*}(t)=\frac{F_{n}(t)}{1+\frac{q-1}{q} \sum_{l} F_{l}(t)\left\langle\widehat{P}_{l}\right\rangle^{t}} .
$$


Множители Лагранжа $F_{n}(t)$ определяются из условий самосогласования:

$$
\left\langle\widehat{P}_{n}\right\rangle^{t}=\left\langle\widehat{P}_{n}\right\rangle_{\text {rel }}^{t}
$$

Для того чтобы найти $\mathrm{HCO}(1)$, необходимо раскрыть явную структуру обобщенного проекционного оператора Кавасаки-Гантона (2). Для этого необходимо рассчитать вариационную производную от релевантного статистического оператоpa $\delta \varrho_{\mathrm{rel}(t)} / \delta\left\langle\widehat{P}_{m}\right\rangle^{t}$, а следовательно, и производную $\delta F_{n}^{*}(t) / \delta\left\langle\widehat{P}_{m}\right\rangle^{t}$, которую можно рассчитать как $\left[\delta\left\langle\widehat{P}_{m}\right\rangle^{t} / \delta F_{n}^{*}(t)\right]^{-1}$. Тогда действие операторов $P_{\mathrm{rel}}(t) i L_{N}$ на peлевантный статистический оператор можно представить в виде $P_{\text {rel }}(t) i L_{N} \varrho_{\text {rel }}(t)=$ $P_{\text {rel }}(t) A(t) \varrho_{\text {rel }}(t)=[P(t) A(t)] \varrho_{\text {rel }}(t)$, где мы ввели обобщенный проекционный оператор, действующий на динамические переменные:

$$
P(t) \bullet=\sum_{m, n}\left\langle\cdot \widehat{P}_{m}\right\rangle_{\mathrm{rel}}\left\langle\widehat{P}_{m} \delta\left\{[q \psi(t)]^{-1} \widehat{P}_{n}\right\}\right\rangle_{\mathrm{rel}}^{-1} \delta\left\{[q \psi(t)]^{-1} \widehat{P}_{n}\right\}
$$

где

$$
\psi(t)=1-\frac{q-1}{q} \sum_{n} F_{n}^{*}(t) \widehat{P}_{n}
$$

Учитывая, что

$$
\left[1-P_{\text {rel }}(t)\right] i L_{N} \varrho_{\text {rel }}(t)=-\sum_{n} I_{n}(t) F_{n}(t) \varrho_{\text {rel }}(t)
$$

с обобщенными потоками

$$
I_{n}(t)=[1-P(t)] \frac{1}{q} \psi^{-1}(t) \dot{\widehat{P}}_{n},
$$

мы можем записать НСО в явном виде [79]:

$$
\varrho\left(x^{N} ; t\right)=\varrho_{\text {rel }}\left(x^{N} ; t\right)-\sum_{n} \int_{-\infty}^{t} e^{\varepsilon\left(t^{\prime}-t\right)} T\left(t, t^{\prime}\right) I_{n}\left(t^{\prime}\right) F_{n}\left(t^{\prime}\right) \varrho_{\text {rel }}\left(x^{N} ; t^{\prime}\right) d t^{\prime} .
$$

С его помощью можно получить обобщенные уравнения переноса для параметров сокращенного описания, которые мы представим следующим образом:

$$
\frac{\partial}{\partial t}\left\langle\widehat{P}_{m}\right\rangle^{t}=\left\langle\dot{\widehat{P}}_{m}\right\rangle_{\mathrm{rel}}^{t}+\sum_{n} \int_{-\infty}^{t} e^{\varepsilon\left(t^{\prime}-t\right)} \varphi_{m n}\left(t, t^{\prime}\right) F_{n}\left(t^{\prime}\right) d t^{\prime} .
$$

Первый член в правой части уравнения рассчитывается с помощью релевантного статистического оператора (5). Обобщенные ядра переноса (функции памяти)

$$
\varphi_{m n}\left(t, t^{\prime}\right)=\int d \Gamma_{N} \dot{\widehat{P}}_{m} T\left(t, t^{\prime}\right) I_{n}\left(t^{\prime}\right) \varrho_{\text {rel }}\left(t^{\prime}\right)
$$

представляют собой временны́е корреляционные функции, которые описывают диссипативные процессы в системе и построены на обобщенных потоках $I_{n}(t)$. В случае $q=1$ приходим к соотношениям, известным в статистике Гиббса. Уравнения переноса (10), в общем случае учитывающие эффекты памяти, в приближении $\varphi_{m n}\left(t, t^{\prime}\right) \approx \varphi_{m n} \delta\left(t-t^{\prime}\right)$ описывают марковские процессы. НСО (9) и уравнения 
переноса (10) в статистике Реньи составляют полный набор инструментов для описания неравновесных процессов в системах, далеких от равновесия, когда определены параметры сокращенного описания $\left\langle\widehat{P}_{n}\right\rangle^{t}$.

В следующем разделе на основании представленного подхода получим уравнения переноса в пространственно-временнь́х дробных производных для классической системы с фрактальностью.

\section{3. УРАВНЕНИЕ ЛИУВИЛЛЯ В ДРОБНЫХ ПРОИЗВОДНЫХ ДЛЯ КЛАССИЧЕСКОЙ СИСТЕМЫ ЧАСТИЦ}

В исследованиях явлений аномальной диффузии в пористых средах [95]-[99], в неупорядоченных системах [87], [100]-[104], физике плазмы [105]-[107], турбулентных [83], [108], кинетических и реакционно-диффузионных процессах [95], [109] активно используются уравнения переноса в дробных производных [95], [110], [111]. Важным является статистическое обоснование подходов к получению уравнений переноса в дробных производных. В работах [112]-[116] было предложено уравнение Лиувилля в дробных производных, а также получены цепочка уравнений Боголюбова-Борна-Грина-Кирквуда-Ивона и уравнения гидродинамики.

Будем исходить из уравнения Лиувилля в дробных производных для неравновесной функции распределения частиц $\varrho\left(x^{N} ; t\right)$ классической системы, предложенного Тарасовым [112]-[115]:

$$
\frac{\partial}{\partial t} \varrho\left(x^{N} ; t\right)+\sum_{j=1}^{N} D_{\vec{r}_{j}}^{\alpha}\left(\varrho\left(x^{N} ; t\right) \vec{v}_{j}\right)+\sum_{j=1}^{N} D_{\vec{p}_{j}}^{\alpha}\left(\varrho\left(x^{N} ; t\right) \vec{F}_{j}\right)=0,
$$

где $x^{N}=x_{1}, \ldots, x_{N}, x_{j}=\left\{\vec{r}_{j}, \vec{p}_{j}\right\}$ - размерные обобщенные координаты $\vec{r}_{j}=r_{j 1} \cdots r_{j l}$ и размерные обобщенные импульсы $\vec{p}_{j}=p_{j 1} \cdots p_{j l} j$-й частицы в фазовом пространстве [116] с фрактальным дифференциальным элементом объема $d^{\alpha} V=d^{\alpha} x_{1} \cdots d^{\alpha} x_{N}$ [112], [117]. Здесь $m=M r_{0} / p_{0} t_{0}, M$ - масса частицы, $r_{0}$ - характерная длина в конфигурационном пространстве, $p_{0}$ - характерное значение импульса, $t_{0}$ - характерное время. Фрактальный дифференциал $d^{\alpha}$ [117] определен следующим образом:

$$
d^{\alpha} f(x)=\sum_{j=1}^{2 N} D_{x_{j}}^{\alpha} f(x)\left(d x_{j}\right)^{\alpha}
$$

где

$$
D_{x}^{\alpha} f(x)=\frac{1}{\Gamma(n-\alpha)} \int_{0}^{x} \frac{f^{(n)}(z)}{(x-z)^{\alpha+1-n}} d z
$$

- фрактальная производная Капуто [110], [118], [119] (здесь $f^{(n)}(z)=d^{n} f(z) / d z^{n}$, $n-1<\alpha<n)$ со следующими свойствами: $D_{x_{j}}^{\alpha} 1=0$ и $D_{x_{j}}^{\alpha} x_{l}=0, j \neq l$. В общем случае

$$
D_{\vec{r}_{j}}^{\alpha}\left(\varrho\left(x^{N} ; t\right) \vec{F}_{j}\right) \neq \varrho\left(x^{N} ; t\right) D_{\vec{r}_{j}}^{\alpha} \vec{F}_{j}+\vec{F}_{j} D_{\vec{r}_{j}}^{\alpha} \varrho\left(x^{N} ; t\right) .
$$

Если обобщенные силы $\vec{F}_{j}$ не зависят от $\vec{p}_{j}$, а обобщенные скорости $\vec{v}_{j}$ не зависят от $\vec{r}_{j}$ и выполняются условия Гельмгольца

$$
\frac{\partial v_{j}}{\partial p_{l}}-\frac{\partial v_{l}}{\partial p_{j}}=0, \quad \frac{\partial v_{j}}{\partial r_{l}}+\frac{\partial F_{l}}{\partial p_{j}}=0, \quad \frac{\partial F_{j}}{\partial r_{l}}-\frac{\partial F_{l}}{\partial r_{j}}=0,
$$


то из (12) мы получаем уравнение Лиувилля в следующем виде:

$$
\frac{\partial}{\partial t} \varrho\left(x^{N} ; t\right)+i L_{\alpha} \varrho\left(x^{N} ; t\right)=0 .
$$

Здесь $i L_{\alpha}$ - оператор Лиувилля в дробных производных [120]:

$$
i L_{\alpha} \varrho\left(x^{N} ; t\right)=\left(\sum_{j=1}^{N} D_{\vec{p}_{j}}^{\alpha} H(\vec{r}, \vec{p}) D_{\vec{r}_{j}}^{\alpha}-\sum_{j=1}^{N} D_{\vec{r}_{j}}^{\alpha} H(\vec{r}, \vec{p}) D_{\vec{p}_{j}}^{\alpha}\right) \varrho\left(x^{N} ; t\right),
$$

а $H(\vec{r}, \vec{p})$ - гамильтониан системы в дробных производных.

Решение уравнения Лиувилля (14) в методе НСО Зубарева будет иметь вид (1) с соответствующим оператором Лиувилля $i L_{\alpha}(15)$. Релевантный статистический оператор $\varrho_{\text {rel }}\left(x^{N} ; t^{\prime}\right)$ будем строить также на основании подхода [79] из экстремума функционала энтропии Реньи при фиксированных значениях наблюдаемых величин $\left\langle\widehat{P}_{n}(x)\right\rangle_{\alpha}^{t}$ и сохранении условия нормировки $\langle 1\rangle_{\alpha, \mathrm{rel}}^{t}=1$. При этом неравновесные средние значения определяются соответственно [120]:

$$
\left\langle\widehat{P}_{n}(x)\right\rangle_{\alpha}^{t}=\widehat{I}^{\alpha}(1, \ldots, N) \widehat{T}(1, \ldots, N) \widehat{P}_{n} \varrho\left(x^{N} ; t\right) .
$$

Для системы $N$ частиц оператор $\widehat{I}^{\alpha}(1, \ldots, N)$ имеет следующий вид:

$$
\widehat{I}^{\alpha}(1, \ldots, N)=\widehat{I}^{\alpha}(1) \ldots \widehat{I}^{\alpha}(N), \quad \widehat{I}^{\alpha}(j)=\widehat{I}^{\alpha}\left(\vec{r}_{j}\right) \widehat{I}^{\alpha}\left(\vec{p}_{j}\right)
$$

и определяет операцию интегрирования

$$
\widehat{I}^{\alpha}(x) f(x)=\int_{-\infty}^{\infty} f(x) d \mu_{\alpha}(x), \quad d \mu_{\alpha}(x)=\frac{|x|^{\alpha}}{\Gamma(\alpha)} d x .
$$

Действие оператора $\widehat{T}(1, \ldots, N)=\widehat{T}(1) \cdots \widehat{T}(N)$ определяется правилом

$$
\widehat{T}\left(x_{j}\right) f\left(x_{j}\right)=\frac{1}{2}\left[f\left(\ldots, x_{j}^{\prime}-x_{j}, \ldots\right)+f\left(\ldots, x_{j}^{\prime}+x_{j}, \ldots\right)\right] .
$$

Средние значения, вычисляемые с релевантным статистическим оператором, определяются как

$$
\langle(\bullet)\rangle_{\alpha, \text { rel }}^{t}=\widehat{I}^{\alpha}(1, \ldots, N) \widehat{T}(1, \ldots, N)(\bullet) \varrho_{\text {rel }}\left(x^{N} ; t\right) .
$$

Тогда релевантный статистический оператор имеет следующий вид [120]:

$$
\varrho_{\text {rel }}(t)=\frac{1}{Z_{\mathrm{R}}(t)}\left[1-\frac{q-1}{q} \sum_{n} \int d \mu_{\alpha}(x) F_{n}^{*}(x ; t) \widehat{P}_{n}(x)\right]^{1 /(q-1)} .
$$

В этом выражении $Z_{\mathrm{R}}(t)$ - статистическая сумма распределения Реньи, которая определяется из условия нормировки и имеет вид

$$
Z_{\mathrm{R}}(t)=\widehat{I}^{\alpha}(1, \ldots, N) \widehat{T}(1, \ldots, N)\left\{1-\frac{q-1}{q} \sum_{n} \int d \mu_{\alpha}(x) F_{n}^{*}(x ; t) \widehat{P}_{n}(x)\right\}^{1 /(q-1)}
$$


где

$$
F_{n}^{*}\left(x ; t^{\prime}\right)=\frac{F_{n}\left(x ; t^{\prime}\right)}{1+\frac{q-1}{q} \sum_{n} \int d \mu_{\alpha}(x) F_{n}\left(x ; t^{\prime}\right)\left\langle P_{n}(x)\right\rangle_{\alpha}^{t}},
$$

а параметры $F_{n}(x ; t)$ определяются из условий самосогласования

$$
\left\langle\widehat{P}_{n}(x)\right\rangle_{\alpha}^{t}=\left\langle\widehat{P}_{n}(x)\right\rangle_{\alpha, \mathrm{rel}}^{t} .
$$

В общем случае для параметров сокращенного описания $\left\langle\widehat{P}_{n}(x)\right\rangle_{\alpha}^{t}$ неравновесных процессов согласно (1) и (18) получаем НСО в виде

$$
\varrho(t)=\varrho_{\mathrm{rel}}(t)+\sum_{n} \int d \mu_{\alpha}(x) \int_{-\infty}^{t} e^{\varepsilon\left(t^{\prime}-t\right)} T\left(t, t^{\prime}\right) I_{n}\left(x ; t^{\prime}\right) \varrho_{\mathrm{rel}}\left(t^{\prime}\right) F_{n}^{*}\left(x ; t^{\prime}\right) d t^{\prime},
$$

где

$$
I_{n}\left(x ; t^{\prime}\right)=[1-P(t)] \frac{1}{q} \psi^{-1}(t) i L_{\alpha} \widehat{P}_{n}(x)
$$

- обобщенные потоки, $P(t)$ - проекционный оператор Мори [120]. Функция $\psi(t)$ имеет следующую структуру:

$$
\psi(t)=1-\frac{q-1}{q} \sum_{n} \int d \mu_{\alpha}(x) F_{n}(x ; t) P_{n}(x) .
$$

С помощью $\mathrm{HCO}(21)$ можно получить обобщенные уравнения переноса для параметров сокращенного описания $\left\langle\widehat{P}_{n}(x)\right\rangle_{\alpha}^{t}$ в виде

$$
\begin{aligned}
\frac{\partial}{\partial t}\left\langle\widehat{P}_{n}(x)\right\rangle_{\alpha}^{t}=\left\langle i L_{\alpha} \widehat{P}_{n}(x)\right\rangle_{\alpha, \mathrm{rel}}^{t}+ & \\
& +\sum_{n^{\prime}} \int d \mu_{\alpha}\left(x^{\prime}\right) \int_{-\infty}^{t} e^{\varepsilon\left(t^{\prime}-t\right)} \varphi_{P_{n} P_{n^{\prime}}}\left(x, x^{\prime} ; t, t^{\prime}\right) F_{n^{\prime}}^{*}\left(x^{\prime} ; t^{\prime}\right) d t^{\prime},
\end{aligned}
$$

где

$$
\begin{aligned}
& \varphi_{P_{n} P_{n^{\prime}}}\left(x, x^{\prime} ; t, t^{\prime}\right)= \\
& \quad=\widehat{I}^{\alpha}(1, \ldots, N) \widehat{T}(1, \ldots, N)\left[i L_{\alpha} \widehat{P}_{n}(x) T\left(t, t^{\prime}\right) I_{n^{\prime}}\left(x^{\prime} ; t^{\prime}\right) \varrho_{\text {rel }}\left(x^{N} ; t^{\prime}\right)\right]
\end{aligned}
$$

- обобщенные ядра переноса (функции памяти), которые описывают диссипативные процессы в системе. В частности, для описания диффузионных процессов в классических системах частиц в среде с фрактальной структурой при постоянной температуре параметром сокращенного описания является неравновесная плотность числа частиц $n(\vec{r} ; t)=\langle\hat{n}(\vec{r})\rangle_{\alpha}^{t}$, где $n(\vec{r})=\sum_{j=1}^{N} \delta\left(\vec{r}-\vec{r}_{j}\right)$ - микроскопическая плотность числа частиц. В этом случае на основании соотношений (18), (21) и уравнений переноса (23) получаем немарковское уравнение диффузии с пространственными дробными производными:

$$
\frac{\partial}{\partial t}\langle\hat{n}(\vec{r})\rangle_{\alpha}^{t}=\frac{\partial^{\alpha}}{\partial \vec{r}^{\alpha}} \int d \mu_{\alpha}\left(\vec{r}^{\prime}\right) \int_{-\infty}^{t} e^{\varepsilon\left(t^{\prime}-t\right)} D_{q}\left(\vec{r}, \vec{r}^{\prime} ; t, t^{\prime}\right) \frac{\partial^{\alpha}}{\partial \vec{r}^{\prime \alpha}} \beta \nu^{*}\left(\vec{r}^{\prime} ; t^{\prime}\right) d t^{\prime} .
$$

Здесь

$$
\begin{aligned}
D_{q}\left(\vec{r}, \vec{r}^{\prime} ; t, t^{\prime}\right)= & \left\langle\hat{\vec{v}}(\vec{r}) T\left(t, t^{\prime}\right) \hat{\vec{v}}\left(\vec{r}^{\prime}\right)\right\rangle_{\alpha, \mathrm{rel}}^{t}= \\
= & \widehat{I}^{\alpha}(1, \ldots, N) \widehat{T}(1, \ldots, N) \hat{\vec{v}}(\vec{r}) T\left(t, t^{\prime}\right) \hat{\vec{v}}\left(\vec{r}^{\prime}\right) \times \\
& \quad \times \frac{1}{Z_{\mathrm{R}}(t)}\left\{1-\frac{q-1}{q} \beta\left[H-\int d \mu_{\alpha}(\vec{r}) \nu^{*}(\vec{r} ; t) \hat{n}(\vec{r})\right]\right\}^{1 /(q-1)}
\end{aligned}
$$


- обобщенный коэффициент диффузии в статистике Реньи, в котором усреднение выполняется со степенным распределением Реньи, где $\hat{\vec{v}}(\vec{r})=\sum_{j=1}^{N} \vec{v}_{j} \delta\left(\vec{r}-\vec{r}_{j}\right)$ - микроскопическая плотность потока частиц, $\beta=1 / k T, k$ - постоянная Больцмана, $T-$ равновесная температура. При $q=1$ обобщенное уравнение диффузии в статистике Реньи переходит в обобщенное уравнение диффузии статистики Гиббса в дробных производных. Когда же $q=1$ и $\alpha=1$, мы приходим к обобщенному уравнению диффузии статистики Гиббса [5]. Пренебрегая пространственной неоднородностью для обобщенного коэффициента диффузии в марковском приближении во времени, можно записать $D_{q}\left(\vec{r}, \vec{r}^{\prime} ; t, t^{\prime}\right) \approx D_{q} \delta\left(t-t^{\prime}\right) \delta\left(\vec{r}-\vec{r}^{\prime}\right)$. Исключив параметр $\nu^{*}\left(\vec{r}^{\prime} ; t^{\prime}\right)$ при помощи условия самосогласования, из (25) получаем уравнение диффузии в дробных производных:

$$
\frac{\partial}{\partial t}\langle\hat{n}(\vec{r})\rangle_{\alpha}^{t}=D_{q} \frac{\partial^{2 \alpha}}{\partial r^{2 \alpha}}\langle\hat{n}(\vec{r})\rangle_{\alpha}^{t}
$$

Обобщенное уравнение диффузии учитывает пространственную фрактальность системы и эффекты памяти в обобщенном коэффициенте диффузии частиц $D_{q}\left(\vec{r}, \vec{r}^{\prime} ; t, t^{\prime}\right)$ в статистике Реньи. Пространственная фрактальность системы, очевидно, влияет на процессы переноса частиц, которые связаны с характерными временами релаксации. Известно, что неравновесные корреляционные функции $D_{q}\left(\vec{r}, \vec{r}^{\prime} ; t, t^{\prime}\right)$ невозможно рассчитать точно, поэтому используют конкретные приближения исходя из физических соображений. Во временно́м интервале $-\infty \div t$ процессы переноса частиц в пространственно неоднородной системе с фрактальной структурой могут характеризоваться совокупностью времен релаксации, которые связаны с характером взаимодействия частиц со средой. Для раскрытия временно́й мультифрактальности в обобщенном уравнении диффузии используем следующее приближение для обобщенного коэффициента диффузии частиц:

$$
D_{q}\left(\vec{r}, \vec{r}^{\prime} ; t, t^{\prime}\right)=W\left(t, t^{\prime}\right) \bar{D}_{q}\left(\vec{r}, \vec{r}^{\prime}\right),
$$

в котором $W\left(t, t^{\prime}\right)$ можно определить как функцию памяти во времени. С учетом этого соотношения уравнение (25) можно представить в виде

$$
\frac{\partial}{\partial t}\langle\hat{n}(\vec{r})\rangle_{\alpha}^{t}=\int_{-\infty}^{t} e^{\varepsilon\left(t^{\prime}-t\right)} W\left(t, t^{\prime}\right) \Psi\left(\vec{r} ; t^{\prime}\right) d t^{\prime},
$$

где

$$
\Psi\left(\vec{r} ; t^{\prime}\right)=\int d \mu_{\alpha}\left(\vec{r}^{\prime}\right) \frac{\partial^{\alpha}}{\partial \vec{r}^{\alpha}} \bar{D}_{q}\left(\vec{r}, \vec{r}^{\prime}\right) \frac{\partial^{\alpha}}{\partial \vec{r}^{\prime \alpha}} \beta \nu^{*}\left(\vec{r}^{\prime} ; t^{\prime}\right) .
$$

Далее, применив преобразование Фурье к уравнению (29), получим

$$
i \omega n(\vec{r} ; \omega)=W(\omega) \Psi(\vec{r} ; \omega) .
$$

Зависимость функции памяти от частоты представим выражением, в котором введено время релаксации $\tau$, характеризующее процессы переноса частиц в системе:

$$
W(\omega)=\frac{(i \omega)^{1-\gamma}}{1+i \omega \tau}, \quad 0 \leqslant \gamma<1 .
$$

Тогда уравнение (31) можно записать в виде

$$
(1+i \omega \tau) i \omega n(\vec{r} ; \omega)=(i \omega)^{1-\gamma} \Psi(\vec{r} ; \omega) .
$$


Применим преобразование Фурье к дробным производным от функций:

$$
L\left({ }_{0} D_{t}^{1-\gamma} f(t) ; i \omega\right)=(i \omega)^{(1-\gamma)} L(f(t) ; i \omega),
$$

где ${ }_{0} D_{t}^{1-\gamma}$ - оператор дробного дифференцирования Римана-Лиувилля [110]:

$$
{ }_{0} D_{t}^{1-\gamma} f(t)=\frac{1}{\Gamma(\gamma)} \frac{d}{d t} \int_{0}^{t} \frac{f\left(t^{\prime}\right)}{\left(t-t^{\prime}\right)^{1-\gamma}} d t^{\prime},
$$

с использованием которого обратное преобразование к временно́й зависимости в уравнении (33) дает обобщенное уравнение диффузии типа Кеттано с учетом пространственной и временно́й фрактальности:

$$
\tau \frac{\partial^{2}}{\partial t^{2}} n(\vec{r} ; t)+\frac{\partial}{\partial t} n(\vec{r} ; t)={ }_{0} D_{t}^{1-\gamma} \int d \mu_{\alpha}\left(\vec{r}^{\prime}\right) \frac{\partial^{\alpha}}{\partial \vec{r}^{\alpha}} \bar{D}_{q}\left(\vec{r}, \vec{r}^{\prime}\right) \frac{\partial^{\alpha}}{\partial \vec{r}^{\prime \alpha}} \beta \nu^{*}\left(\vec{r}^{\prime} ; t\right) .
$$

Важно отметить, что если в уравнении (35) положить $\alpha=1$, т. е. пренебречь пространственной фрактальностью, а также пространственной неоднородностью $\bar{D}_{q}\left(\vec{r}, \vec{r}^{\prime}\right)=\bar{D}_{q} \delta\left(\vec{r}-\vec{r}^{\prime}\right)$, то при $q=1$ (в случае статистики Гиббса) получим уравнение диффузии типа Кеттано, которое ранее было получено в работах [99], [121],

$$
\tau \frac{\partial^{2}}{\partial t^{2}} n(\vec{r} ; t)+\frac{\partial}{\partial t} n(\vec{r} ; t)={ }_{0} D_{t}^{1-\gamma} \bar{D} \frac{\partial^{2}}{\partial \vec{r}^{2}} \nu(\vec{r} ; t) .
$$

Это уравнение было успешно использовано для исследований субдиффузионного импеданса в мультислоистых наноструктурах [122].

\section{4. НЕРАВНОВЕСНАЯ ТЕРМОПОЛЕВАЯ ДИНАМИКА В СТАТИСТИКЕ РЕНЬИ}

В современных теоретических исследованиях неравновесных свойств кварк-глюонной плазмы [123]-[125] - одного из состояний ядерной материи, которая может образовываться в ультрарелятивистских столкновениях тяжелых ядер [126], - активно используются статистические подходы с применением определений энтропии Цаллиса и Реньи [127]-[135]. При этом важной проблемой является построение кинетических уравнений и уравнений гидродинамики для ядерной материи большой плотности и высокой температуры. Одним из возможных подходов к решению этих задач может быть неравновесная термополевая динамика.

Мы будем использовать метод НСО в термополевой формулировке [59], в которой средние значения величин, соответствующих наблюдаемым переменным, находятся с помощью неравновесного термовакуумного вектора состояния $|\varrho(t)\rangle\rangle$ :

$$
\langle A\rangle^{t}=\langle\langle 1 \mid A \varrho(t)\rangle\rangle=\langle\langle 1|\widehat{A}| \varrho(t)\rangle\rangle
$$

где $\widehat{A}$ - супероператор, который действует на состояние $|\varrho(t)\rangle$. Неравновесный термовакуумный вектор состояния $|\varrho(t)\rangle\rangle$ удовлетворяет "уравнению Шредингера" [59]:

$$
\left.\left.\frac{\partial}{\partial t}|\varrho(t)\rangle\right\rangle-\left|\frac{1}{i \hbar}[H, \varrho(t)]\right\rangle\right\rangle=0
$$

или

$$
\left.\left.\frac{\partial}{\partial t}|\varrho(t)\rangle\right\rangle-\frac{1}{i \hbar} \widehat{H}|\varrho(t)\rangle\right\rangle=0
$$


Здесь "полный" гамильтониан $\widehat{H}$ имеет вид

$$
\widehat{H}=H-\widetilde{H}
$$

причем $\left\langle\langle 1| \widehat{H}=0\right.$, а $H=H\left(\hat{a}^{+}, \hat{a}\right), \widetilde{H}=H^{(*)}\left(\tilde{a}^{+}, \tilde{a}\right)$ - супероператоры, построенные на супероператорах рождения и уничтожения термического пространства Лиувилля [136]-[138]. Супероператоры $H$ и $\widetilde{H}$ определены согласно соотношениям

$$
|H \varrho(t)\rangle\rangle=H|\varrho(t)\rangle\rangle, \quad|\varrho(t) H\rangle\rangle=\widetilde{H}|\varrho(t)\rangle\rangle .
$$

Супероператоры $\hat{a}_{l}^{+}, \hat{a}_{j}, \tilde{a}_{l}^{+}, \tilde{a}_{j}$ удовлетворяют тем же коммутационным соотношениям, что и операторы $a_{l}^{+}, a_{j}$ соответствующей статистики:

$$
\begin{array}{rlrl}
{\left[\hat{a}_{l}, \hat{a}_{j}^{+}\right]_{\sigma}} & =\left[\tilde{a}_{l}, \tilde{a}_{j}^{+}\right]_{\sigma}=\delta_{l j}, & {\left[\hat{a}_{l}, \tilde{a}_{j}\right]_{\sigma}=\left[\hat{a}_{l}^{+}, \tilde{a}_{j}^{+}\right]_{\sigma}=0,} \\
{\left[\hat{a}_{l}, \hat{a}_{j}\right]_{\sigma}=\left[\hat{a}_{l}^{+}, \hat{a}_{j}^{+}\right]_{\sigma}=0,} & {\left[\tilde{a}_{l}, \tilde{a}_{j}\right]_{\sigma}=\left[\tilde{a}_{l}^{+}, \tilde{a}_{j}^{+}\right]_{\sigma}=0,}
\end{array}
$$

где $[A, B]_{\sigma}=A B-\sigma B A$, а $\sigma=+1$ для бозонов и $\sigma=-1$ для фермионов. Супероператоры уничтожения $\hat{a}_{l}, \tilde{a}_{l}$ определяются согласно их действию на основное состояние - супервакуум [139]-[141]:

$$
\left.\left.\hat{a}_{l}|00\rangle\right\rangle=\tilde{a}_{l}|00\rangle\right\rangle=0,
$$

где $|00\rangle\rangle=|0\rangle\langle 0 \mid\rangle\rangle$ - супервакуум. Причем имеют место соотношения $\hat{a}_{l}|0\rangle=a_{l}|0\rangle=$ 0 и $\langle 0| \tilde{a}_{l}=0$, т.е. супервакуум $\left.|00\rangle\right\rangle$ является ортогонализированным состоянием двух вакуумных состояний $\langle 0|$ и $|0\rangle$. Учитывая коммутационные соотношения (42) и определения (43), единичные векторы $\left.\left.|1\rangle\rangle=\left|\sum_{l}\right| l\right\rangle\langle l \mid\rangle\right\rangle$ и $\left\langle\langle 1|=\left\langle\left\langle\sum_{l} \mid l\right\rangle\langle l||\right.\right.$ можно представить в виде

$$
\left.|1\rangle\rangle=\exp \left\{\sum_{l} \hat{a}_{l}^{+} \tilde{a}_{l}^{+}\right\}|00\rangle\right\rangle, \quad\left\langle\langle 1|=\left\langle\langle 00| \exp \left\{\sum_{l} \tilde{a}_{l} \hat{a}_{l}\right\} .\right.\right.
$$

С их помощью этих векторов найдем соотношения между действиями супероператоров $\hat{a}_{l}^{+}, \hat{a}_{j}, \tilde{a}_{l}^{+}, \tilde{a}_{j}$ :

$$
\begin{aligned}
\left.\hat{a}_{l}|1\rangle\right\rangle & \left.=\tilde{a}_{l}^{+}|1\rangle\right\rangle, \quad & \left\langle\langle 1| \hat{a}_{l}^{+}\right. & =\left\langle\langle 1| \tilde{a}_{l},\right. \\
\left.\hat{a}_{l}^{+}|1\rangle\right\rangle & \left.=\sigma \tilde{a}_{l}|1\rangle\right\rangle, & \left\langle\langle 1| \hat{a}_{l}\right. & =\left\langle\langle 1| \tilde{a}_{l}^{+} \sigma .\right.
\end{aligned}
$$

Таким образом, в формализме термополевой динамики [139]-[141] число операторов удвоено введением операторов $A\left(\hat{a}^{+}, \hat{a}\right)$ и $\widetilde{A}\left(\tilde{a}^{+}, \tilde{a}\right)$, для которых выполняются следующие свойства:

$$
\begin{gathered}
\widetilde{A_{1} A_{2}}=\widetilde{A}_{1} \widetilde{A}_{2}, \quad \widetilde{\widetilde{A}}=A, \\
c_{1} \widetilde{A_{1}+c_{2}} A_{2}=c_{1}^{*} \widetilde{A}_{1}+c_{2}^{*} \widetilde{A}_{2}, \\
\left.\left.|A\rangle\rangle=\widehat{A}|1\rangle\rangle, \quad\left|A_{1} A_{2}\right\rangle\right\rangle=\widehat{A}_{1}\left|A_{2}\right\rangle\right\rangle,
\end{gathered}
$$

где звездочка обозначает комплексное сопряжение. Более подробное изложение свойств супероператоров $\hat{a}_{l}^{+}, \hat{a}_{j}, \tilde{a}_{l}^{+}, \tilde{a}_{j}$ и термического пространства Лиувилля дано в работах [139]-[141]. Неравновесный термовакуумный вектор состояния нормирован: $\langle\langle 1 \mid \varrho(t)\rangle\rangle=\langle\langle 1|\varrho(t)| 1\rangle\rangle=1$, где $\varrho(t)-$ неравновесный статистический супероператор, который зависит от супероператоров $\hat{a}_{l}^{+}, \hat{a}_{j}, \hat{\varrho}(t) \equiv \varrho\left(\hat{a}^{+}, \hat{a} ; t\right)$. Причем супероператор $\tilde{\varrho}(t) \equiv \varrho^{+}\left(\tilde{a}^{+}, \tilde{a} ; t\right)$ зависит от супероператоров $\tilde{a}_{l}^{+}, \tilde{a}_{j}$. 
В методе НСО в термополевой формулировке [59], [60] неравновесный термовакуумный вектор состояния, как решение "уравнения Шредингера" с источником

$$
\left.\left.\left.\left(\frac{\partial}{\partial t}-\frac{1}{i \hbar} \widehat{H}\right)|\varrho(t)\rangle\right\rangle=-\varepsilon(|\varrho(t)\rangle\rangle-\left|\varrho_{\text {rel }}(t)\right\rangle\right\rangle\right),
$$

с учетом проецирования может быть найден в виде [59]

$$
\left.\left.|\varrho(t)\rangle\rangle=\left|\varrho_{\text {rel }}(t)\right\rangle\right\rangle+\int_{-\infty}^{t} d t^{\prime} e^{\varepsilon\left(t^{\prime}-t\right)} T\left(t, t^{\prime}\right)\left[1-\mathcal{P}_{\text {rel }}\left(t^{\prime}\right)\right] \frac{1}{i \hbar} \widehat{H}\left|\varrho_{\text {rel }}\left(t^{\prime}\right)\right\rangle\right\rangle .
$$

Здесь

$$
T\left(t, t^{\prime}\right)=\exp _{+}\left\{\int_{t^{\prime}}^{t} d t^{\prime}\left[1-\mathcal{P}_{\text {rel }}\left(t^{\prime}\right)\right] \frac{1}{i \hbar} \widehat{H}\right\}
$$

- оператор эволюции с учетом проецирования, где ехр - $_{+}$упорядоченная экспонента, а $\varepsilon \rightarrow+0$ после термодинамического предельного перехода,

$$
\begin{aligned}
\left.\left.\mathcal{P}_{\text {rel }}(t)(|\cdot\rangle\rangle\right)=\left|\varrho_{\text {rel }}(t)\right\rangle\right\rangle+\sum_{n} \frac{\left.\delta\left|\varrho_{\text {rel }}(t)\right\rangle\right\rangle}{\delta\left\langle\left\langle 1\left|\hat{p}_{n}\right| \varrho(t)\right\rangle\right\rangle}\left\langle\left\langle 1\left|\hat{p}_{n}\right| \cdot\right\rangle\right\rangle- \\
-\sum_{n} \frac{\left.\delta\left|\varrho_{\text {rel }}(t)\right\rangle\right\rangle}{\delta\left\langle\left\langle 1\left|\hat{p}_{n}\right| \varrho(t)\right\rangle\right\rangle}\left\langle\left\langle 1\left|\hat{p}_{n}\right| \cdot\right\rangle\right\rangle\langle\langle 1 \mid \cdot\rangle\rangle
\end{aligned}
$$

- проекционный оператор Кавасаки-Гантона, который действует только на векторы состояния $|\cdot\rangle\rangle$ и обладает следующими операторными свойствами: $\left.\mathcal{P}_{\text {rel }}(t)\left|\varrho\left(t^{\prime}\right)\right\rangle\right\rangle=$ $\left.\left.\left.\left|\varrho_{\text {rel }}(t)\right\rangle\right\rangle, \mathcal{P}_{\text {rel }}(t)\left|\varrho_{\text {rel }}\left(t^{\prime}\right)\right\rangle\right\rangle=\left|\varrho_{\text {rel }}(t)\right\rangle\right\rangle, \mathcal{P}_{\text {rel }}(t) \mathcal{P}_{\text {rel }}\left(t^{\prime}\right)=\mathcal{P}_{\text {rel }}(t)$. Релевантный термовакуумный вектор состояния

$$
\left.\left.\left|\varrho_{\text {rel }}(t)\right\rangle\right\rangle=\hat{\varrho}_{\text {rel }}(t)|1\rangle\right\rangle,
$$

нормированный согласно соотношению $\left\langle\left\langle 1 \mid \varrho_{\text {rel }}(t)\right\rangle\right\rangle=\left\langle\left\langle 1\left|\hat{\varrho}_{\text {rel }}(t)\right| 1\right\rangle\right\rangle=1$, где $\hat{\varrho}_{\text {rel }}(t)-$ релевантный статистический супероператор. Релевантный термовакуумный вектор состояния системы определим следующим образом. Пусть $\left\langle p_{n}\right\rangle^{t}=\left\langle\left\langle 1\left|\hat{p}_{n}\right| \varrho(t)\right\rangle\right\rangle-$ набор наблюдаемых переменных, описывающих неравновесное состояние системы, где $p_{n}$ - операторы, которые построены на операторах рождения $a_{l}^{+}$и уничтожения $a_{l}$. Релевантный статистический оператор $\varrho_{\mathrm{rel}}(t)$ будем определять из экстремума функционала энтропии Реньи

$$
L_{\mathrm{R}}(t)=\frac{1}{1-q} \ln \left\langle\left\langle 1 \mid\left(\left|\varrho^{\prime}(t)\right\rangle\right\rangle\right)^{q}-\alpha\left\langle\left\langle 1 \mid \varrho^{\prime}(t)\right\rangle\right\rangle-\sum_{n} F_{n}^{*}(t)\left\langle\left\langle 1\left|\hat{p}_{n}\right| \varrho^{\prime}(t)\right\rangle\right\rangle\right.
$$

при дополнительном условии, что заданы средние значения $\left\langle p_{n}\right\rangle^{t}$ при сохранении условия нормировки $\langle\langle 1|\hat{\varrho}(t)| 1\rangle\rangle=1$. Параметры Лагранжа $\alpha$ и $F_{n}^{*}(t)$ определяются из условия нормировки и условий самосогласования соответственно:

$$
\langle\cdot\rangle_{\mathrm{rel}}^{t}=\left\langle\left\langle 1|\cdot| \varrho_{\mathrm{rel}}(t)\right\rangle\right\rangle, \quad\left\langle p_{n}\right\rangle^{t}=\left\langle p_{n}\right\rangle_{\mathrm{rel}}^{t}=\left\langle\left\langle 1\left|\hat{p}_{n}\right| \varrho_{\mathrm{rel}}(t)\right\rangle\right\rangle .
$$

Тогда релевантный статистический оператор $\varrho_{\text {rel }}(t)$ имеет вид

$$
\varrho_{\mathrm{rel}}(t)=\frac{1}{Z_{\mathrm{R}}(t)}\left[1-\frac{q-1}{q} \sum_{n} F_{n}^{*}(t) \delta \hat{p}_{n}(t)\right]^{1 /(q-1)},
$$


где $q$ - параметр Реньи, $\delta \hat{p}_{n}(t)=\hat{p}-\left\langle\left\langle 1\left|\hat{p}_{n}\right| \varrho(t)\right\rangle\right.$,

$$
Z_{\mathrm{R}}(t)=\left\langle\left\langle 1 \mid\left[1-\frac{q-1}{q} \sum_{n} F_{n}^{*}(t) \delta \hat{p}_{n}(t)\right]^{1 /(q-1)}\right\rangle\right\rangle
$$

- статистическая сумма. При $q=1$ из (52) получим релевантный статистический оператор, что соответствует статистике Гиббса [59]:

$$
\varrho_{\text {rel }}(t)=\exp \left\{-\Phi(t)-\sum_{n} F_{n}^{*}(t) p_{n}\right\}
$$

где $\Phi(t)=\ln \operatorname{Sp} \exp \left\{-\sum_{n} F_{n}^{*}(t) p_{n}\right\}$ - функционал Масье-Планка. Теперь, подставив (52) в (48), для неравновесного термовакуумного вектора состояния получим

$$
\left.\left.|\varrho(t)\rangle\rangle=\left|\varrho_{\mathrm{rel}}(t)\right\rangle\right\rangle+\sum_{n} \int_{-\infty}^{t} d t^{\prime} e^{\varepsilon\left(t^{\prime}-t\right)} T\left(t, t^{\prime}\right)\left|\int_{0}^{1} d \tau \varrho_{\mathrm{rel}}^{\tau}\left(t^{\prime}\right) J_{n}\left(t^{\prime}\right) \varrho_{\mathrm{rel}}(t)^{1-\tau}\left(t^{\prime}\right)\right\rangle\right\rangle F_{n}^{*}\left(t^{\prime}\right),
$$

где $J_{n}(t)=[1-\mathcal{P}(t)](1 / q) \psi^{-1}(t) \dot{\hat{p}}_{n}$ - операторы обобщенных потоков, которые описывают диссипативные процессы в системе, $\dot{\hat{p}}_{n}=-(1 / i \hbar) \widehat{H} \hat{p}_{n}$. Проекционный оператор $\mathcal{P}(t)$ действует на операторы и имеет следующую структуру:

$$
\begin{aligned}
\mathcal{P}(t)(\bullet)= & \left\langle\left\langle 1|\cdot| \varrho_{\text {rel }}(t)\right\rangle+\sum_{m} \delta\left[\int_{0}^{1} d \tau \varrho_{\text {rel }}^{\tau}(t) \psi^{-1}(t) \times\right.\right. \\
& \left.\times\left(F_{m}(t)+\sum_{n} f_{m n}^{-1}(t) \delta \hat{p}_{n}\right) \varrho_{\text {rel }}^{-\tau}\right]\left\langle\left\langle\bullet \mid \int_{0}^{1} d \tau \varrho_{\text {rel }}^{\tau}(t) \delta \hat{p}_{n} \varrho_{\text {rel }}^{-\tau}(t) \varrho_{\text {rel }}(t)\right\rangle\right\rangle,
\end{aligned}
$$

где $\delta[\bullet]=[\bullet]-\left\langle\left\langle 1|[\bullet]| \varrho_{\text {rel }}(t)\right\rangle\right\rangle$ и $f_{m n}(t)=\delta\left\langle\left\langle 1\left|\hat{p}_{m}\right| \varrho(t)\right\rangle\right\rangle / \delta F_{n}(t)$. Оператор $\psi(t)$ имеет вид

$$
\psi(t)=1-\frac{q-1}{q} \sum_{n} F_{n}^{*}(t) \delta \hat{p}_{n}(t) .
$$

С помощью неравновесного термовакуумного вектора состояния $|\varrho(t)\rangle\rangle(55)$ получим уравнения переноса для неравновесных средних значений $\left\langle\left\langle 1\left|\hat{p}_{n}\right| \varrho(t)\right\rangle\right\rangle$ в термополевом представлении. Для этого используем тождество

$$
\frac{\partial}{\partial t}\left\langle\left\langle 1\left|\hat{p}_{n}\right| \varrho(t)\right\rangle\right\rangle=\left\langle\left\langle 1\left|\dot{\hat{p}}_{n}\right| \varrho(t)\right\rangle\right\rangle=\left\langle\left\langle 1\left|\dot{\hat{p}}_{n}\right| \varrho_{\text {rel }}(t)\right\rangle\right\rangle+\left\langle\left\langle J_{n}(t) \mid \varrho(t)\right\rangle\right\rangle
$$

и, усредняя последнее слагаемое в правой части с $|\varrho(t)\rangle\rangle(55)$, получим уравнения переноса для средних значений $\left\langle\left\langle 1\left|\hat{p}_{n}\right| \varrho_{\text {rel }}(t)\right\rangle\right\rangle$ :

$$
\begin{aligned}
& \frac{\partial}{\partial t}\left\langle\left\langle 1\left|\hat{p}_{n}\right| \varrho(t)\right\rangle\right\rangle=\left\langle\left\langle 1\left|\dot{\hat{p}}_{n}\right| \varrho_{\text {rel }}(t)\right\rangle\right\rangle+ \\
& \quad+\sum_{n^{\prime}} \int_{-\infty}^{t} d t^{\prime} e^{\varepsilon\left(t^{\prime}-t\right)}\left\langle\left\langle\dot{\hat{p}}_{n} T\left(t, t^{\prime}\right) \mid \int_{0}^{1} d \tau \varrho_{\text {rel }}^{\tau}\left(t^{\prime}\right) J_{n^{\prime}}\left(t^{\prime}\right) \varrho_{\text {rel }}^{1-\tau}\left(t^{\prime}\right)\right\rangle\right\rangle F_{n^{\prime}}^{*}\left(t^{\prime}\right) .
\end{aligned}
$$

Уравнения переноса (58) учитывают эффекты памяти и могут быть использованы для описания неравновесных процессов в квантовых бозе- и ферми-системах в конкретных случаях в рамках неравновесной термополевой динамики неэкстенсивной 
статистики. В частности, в работе [60] была получена система релятивистских уравнений переноса согласованного описания кинетических и гидродинамических процессов в кварк-глюонной системе с использованием метода НСО в термополевом представлении в статистике Гиббса. Изложенный подход в рамках статистики Реньи может быть обобщен на случай релятивистских систем, что важно [127]-[135] и чему будут посвящены отдельные работы.

\section{5. ЗАКЛЮЧЕНИЕ}

Мы представили обобщение метода НСО Зубарева на основании принципа максимума энтропии Реньи. Применяя этот подход, мы получили уравнения переноса для основного набора параметров сокращенного описания неравновесных процессов в классической системе взаимодействующих частиц, используя уравнения Лиувилля в дробных производных. В частности, для описания диффузионных процессов в классической системе частиц в среде с фрактальной структурой получено немарковское уравнение диффузии с пространственными дробными производными. В случае конкретного моделирования частотной зависимости функции памяти получено обобщенное уравнение диффузии типа Кеттано с учетом пространственной и временно́й фрактальности. Мы обобщили неравновесную термополевую динамику в методе НСО Зубарева [59] в рамках статистики Реньи.

\section{Список литературы}

[1] Д. Н. Зубарев, "Вычисление конфигурационного интеграла системы частиц с кулоновским взаимодействием”, Докл. АН СССР, 95:4 (1954), 757-760.

[2] Д. Н. Зубарев, “Двухвременные функции Грина в статистической физике”, УФН, 71:1 (1960), 71-116.

[3] Д.Н. Зубарев, “Статистический оператор для неравновесных систем”, Докл. АН СCCP, 140:1 (1961), 92-95.

[4] Д. Н. Зубарев, Неравновесная статистическая термодинамика, Наука, М., 1971.

[5] Д.Н. Зубарев, “Современные методы статистической теории неравновесных процессов", Итоги науки и техн. Сер. Соврем. пробл. матем., 15, ВИНИТИ, М., 1980, 131-226.

[6] D. N. Zubarev, "Nonequilibrium statistical operator as a generalization of Gibbs distribution for nonequilibrium case", Condens. Matter Phys., 1994, № 4, 7-25.

[7] V. G. Morozov, G. Röpke, "Zubarev's method of a nonequilibrium statistical operator and some challenges in the theory of irreversible processes", Condens. Matter Phys., 1:4(16) (1998), 673-686.

[8] Д. Н. Зубарев, В. Г. Морозов, Г. Рёпке, Статистическая механика неравновесных процессов, т. 1, Физматлит, M., 2002; англ. пер.: D. N. Zubarev, V. G. Morozov, G. Röpke, Statistical Mechanics of Nonequilibrium Processes, v.1: Basic Concepts, Kinetic Theory, Akademie, Berlin, 1996.

[9] Д. Н. Зубарев, В. Г. Морозов, Г. Рёпке, Статистическая механика неравновесных процессов, т. 2, Физматлит, M., 2002; англ. пер.: D. N. Zubarev, V. G. Morozov, G. Röpke, Statistical Mechanics of Nonequilibrium Processes, v. 2: Relaxation and Hydrodynamic Processes, Akademie, Berlin, 1997.

[10] И.Р. Юхновский, М.Ф. Головко, Статистическая теория классичеких равновесных систем, Наукова думка, Киев, 1980.

[11] И. Р. Юхновский, "Метод коллективных переменных с системой отсчета для большого канонического ансамбля", ТМФ, 79:2 (1989), 282-296. 
[12] I. R. Yukhnovskii, O. V. Patsahan, "Grand canonical distribution for multicomponent system in the collective variables method", J. Statist. Phys., 81:3-4 (1995), 647-672.

[13] І. Р. Юхновський, Вибрані праці. Фізика, Вид. Національного університету “Львівська політехніка", Львів, 2005.

[14] I. R. Yukhnovskii, Phase Transitions of the Second Order. Collective Variables Method, World Sci., Singapore, 1987.

[15] I. R. Yukhnovskii, "The functional of the grand partition function for the investigation of the liquid-gas critical point", Phys. A, 168:3 (1990), 999-1020.

[16] І. Р. Юхновський, М. П. Козловський, І. В. Пилюк, Мікроскопічна теорія фазових переходів у тривимірних системах, Євросвіт, Львів, 2001.

[17] И. А. Вакарчук, И.Р. Юхновский, "Самосогласованное описание дальнодействующих и короткодействующих корреляций в теории жидкого Не ${ }^{4}$. I", TMФ, 40:1 (1979), $100-111$.

[18] И. А. Вакарчук, О. Л. Гонопольский, И. Р. Юхновский, "Самосогласованное описание дальнодействующих и короткодействующих корреляций в теории жидкого $\mathrm{He}^{4}$. II", TMФ, 41:1 (1979), 77-88.

[19] П. П. Костробий, И. Р. Юхновский, "Функции распределения вырожденного электронного газа в периодическом внешнем поле", ТМФ, 32:2 (1977), 208-222.

[20] В. Л. Бонч-Бруевич, С. В. Тябликов, Метод функиий Грина в статистической механике, Физматлит, М., 1961.

[21] Н. М. Плакида, "Метод двухвременных функций Грина в теории ангармонических кристаллов", Статистическая физика и квантовая теория поля, ред. Н. Н. Боголюбов, Наука, М., 1973, 205-240.

[22] Н. М. Плакида, Некоторые вопросы квантовой теории твердого тела (метод двухвременных функиий Грина), Изд-во Моск. ун-та, М., 1974.

[23] Д. Н. Зубарев, Ю. А. Церковников, "Метод двухвременных температурных функций Грина в равновесной и неравновесной статистической механике", Тр. МИАН, 175 (1986), 134-177.

[24] Н. М. Плакида, “Двухвременные функции Грина в теории сверхпроводимости”, ТМФ, 154:1 (2008), 129-146.

[25] Н. М. Плакида, “Двухвременные функции Грина и диаграммная техника”, TMФ, 168:3 (2011), 518-535.

[26] В. Д. Крефт, Д. Кремп, В. Эбелинг, Г. Рёпке, Квантовая статистика систем заряженных частии, Мир, М., 1988.

[27] G. D. Mahan, Many-Particle Physics, Kluwer, New York, 2000.

[28] В. Т. Швець, Метод функиій Гріна в теоріӥ металів, Латстар, Одесса, 2002.

[29] R. Luzzi, A.R. Vasconcellos, J. G. Ramos, Predictive Statistical Mechanics. A Nonequilibrium Ensemble Formalism, Fundamental Theories of Physics, 122, Kluwer, Dordrecht, 2002.

[30] Ю.А. Церковников, Статистическал механика. Избранные труды, Янус-К, М., 2010.

[31] І. В. Стасюк, Функцї Гріна у квантовій статистиці твердих тіл: посібник, Львівський національний університет імені Івана Франко, Львів, 2013.

[32] R. Luzzi, A. R. Vasconcellos, J. G. Ramos, Statistical Foundations of Irreversible Thermodynamics, Springer Science and Business Media, Berlin, 2013.

[33] И. И. Ляпилин, В.П. Калашников, Неравновесный статистический оператор и его приложения к кинетике парамагнитных явлений в проводящих кристаллах, УрО РАН, Екатеринбург, 2008.

[34] Г. Рёпке, Неравновесная статистическая механика, Мир, М., 1990; англ. пер.: G. Röpke, Nonequilibrium Statistical Physics, Wiley-VCH, New York, 2013. 
[35] А.А. Хамзин, Р.Р. Нигматулин, Метод неравновесного статистического оператора и его приложение к кинетике изинговских магнетиков, Учебное пособие, Казан. ун-т, Казань, 2011.

[36] И. М. Мрыглод, М. В. Токарчук, "К статистической гидродинамике простых жидкостей", Вопросы атомной науки и техники, 3(24) (1992), 134-139.

[37] I. M. Mryglod, I. P. Omelyan, M. V. Tokarchuk, "Generalized collective modes for the Lennard-Jones fluid", Mol. Phys., 84:2 (1995), 235-259.

[38] Б. Б. Маркив, И.П. Омелян, М.В. Токарчук, "Неравновесный статистический оператор в обобщенной молекулярной гидродинамике жидкостей”, ТМФ, 154:1 (2008), 91-101.

[39] B. B. Markiv, I. P. Omelyan, M. V. Tokarchuk, "Relaxation to the state of molecular hydrodynamics in the generalized hydrodynamics of liquids", Phys. Rev. E, 82:4 (2010), 041202, $11 \mathrm{pp}$.

[40] I. M. Mryglod, "Generalized statistical hydrodynamics of fluids: approach of generalized collective modes", Condens. Matter Phys., 1:4(16) (1998), 753-796.

[41] I. P. Omelyan, I. M. Mryglod, M. V. Tokarchuk, "Dielectric relaxation in dipolar fluids. Generalized mode approach", Condens. Matter Phys., 1:1(13) (1998), 179-200.

[42] I. P. Omelyan, I. M. Mryglod, M. V. Tokarchuk, "Generalized dipolar modes of a Stockmayer fluid in high-order approximations", Phys. Rev. E., 57:6 (1998), 6667-6676.

[43] I. M. Mryglod, M. V. Tokarchuk, R. Folk, "On the hydrodynamic theory of a magnetic liquid I. General description", Phys. A, 220:3-4 (1995), 325-348.

[44] И. М. Мрыглод, М.В. Токарчук, "Статистическая гидродинамика магнитных жидкостей. І. Метод неравновесного статистического оператора”, ТМФ, 115:1 (1998), $132-153$.

[45] Д. Н. Зубарев, М. В. Токарчук, "Неравновесная статистическая гидродинамика ионных систем", ТМФ, 70:2 (1987), 234-254.

[46] B. Markiv, A. Vasylenko, M. Tokarchuk, "Statistical description of hydrodynamic processes in ionic melts while taking into account polarization effects", J. Chem. Phys., 136:23 (2012), 234502, 10 pp.

[47] B. Markiv, M. Tokarchuk, "Effect of ion polarization on longitudinal excitations in ionic melts", J. Chem. Phys., 143:19 (2015), 194509, 8 pp.

[48] М. В. Токарчук, "К статистической теории неравновесной плазмы в собственном электромагнитном поле", ТМФ, 97:1 (1993), 27-43.

[49] B. Markiv, M. Tokarchuk, "Consistent description of kinetics and hydrodynamics of dusty plasma", Phys. Plasmas, 21:2 (2014), 023707, 16 pp.

[50] В. В. Игнатюк, И. М. Мрыглод, М. В. Токарчук, "К теории динамических свойств полуквантового гелия", ФНТ, 25:5 (1999), 407-416.

[51] В. В. Игнатюк, И. М. Мрыглод, М. В. Токарчук, "Временные корреляционные функции и обобщенные коэффициенты переноса полуквантового гелия”, ФНT, 25:11 (1999), $1145-1153$.

[52] П. П. Костробій, М. В. Токарчук, Б. М. Маркович, В. В. Ігнатюк, Б. В. Гнатів, Реакиійно-дифузійні процеси в системах "метал-газ", Видав. НУ "Львівська політехніка", Львів, 2009.

[53] Д. Н. Зубарев, А. В. Прозоркевич, С. А. Смолянский, "Вывод нелинейных обобщенных уравнений квантовой релятивистской гидродинамики", ТМФ, 40:3 (1979), 394-407.

[54] А. В. Прозоркевич, В. Л. Самородов, С. А. Смолянський, "Квантовая релятивистская гидродинамика систем с нарушенной симметрией. І. Локально-равновесное состояние", TMФ, 52:3 (1982), 463-472.

[55] С. А. Смолянський, "Квантовая релятивистская гидродинамика систем с нарушенной симметрией. II. Неравновесное состояние", ТМФ, 52:2 (1982), 292-300. 
[56] F. Becattini, L. Tinti, "Nonequilibrium thermodynamical inequivalence of quantum stress-energy and spin tensors", Phys. Rev. D, 87:2 (2013), 025029, 15 pp.

[57] L. Tinti, "Thermodynamical inequivalence of stress-energy and spin tensors", Progress in Mathematical Relativity, Gravitation and Cosmology, Proceedings of the Spanish Relativity Meeting ERE2012 (University of Minho, Guimaraes, Portugal, September 3-7, 2012), eds. A. García-Parrado, F. C. Mena, F. Moura, E. Vaz, Springer, Berlin, Heidelberg, 2014, 433-437.

[58] F. Becattini, L. Bucciantini, E. Grossi, L. Tinti, "Local thermodynamical equilibrium and the $\beta$ frame for a quantum relativistic fluid", Eur. Phys. J. C, 75:5 (2015), 191, 17 pp.

[59] Д. Н. Зубарев, М. В. Токарчук, "Неравновесная термополевая динамика и метод неравновесного статистического оператора. І. Основные соотношения", ТМФ, 88:2 (1991), $286-310$.

[60] M. V. Tokarchuk, T. Arimitsu, A. E. Kobryn, "Thermo field hydrodynamic and kinetic equations of dense quantum nuclear systems", Condens. Matter Phys., 1:3(15) (1998), 605-642.

[61] Д.Н. Зубарев, В.Н. Климов, "К теории температурного скачка на границе плазмы в магнитном поле", Физика плазмы и проблема управляемых термоядерных реакиий, т. 1, Изд-во АН СССР, М., 1958, 138-160.

[62] Д. Н. Зубарев, В.Н. Климов, "Стационарные режимы магнитного термоядерного реактора", Физика плазмы и проблема управляемых термоядерных реакций, т. 1, Изд-во AH CCCP, M., 1958, 249-288.

[63] А.В. Недоспасов, "Физика пристеночной плазмы в токамаках", УФН, 152:3 (1987), 479-492.

[64] А. В. Недоспасов, М. З. Токарь, "Пристеночная плазма в токамаках", Вопросы теории плазмы. Вып. 18, ред. Б. Б. Кадомцев, Энергоатомиздат, М., 1990, 68-208.

[65] В. Н. Цытович, "Плазменно-пылевые кристаллы, капли и облака", УФН, 167:1 (1997), 57-99.

[66] В. Н. Цытович, Д. Винтер, “Пыль в установках управляемого термоядерного синтеза", УФН, 168:8 (1998), 899-907.

[67] В.Н. Цытович, “О перспективах экспериментальных и теоретических исследований самоорганизованных пылевых структур в комплексной плазме в условиях микрогравитации", УФН, 185:2 (2015), 161-179.

[68] В.Е. Фортов, А.Г. Храпак, С.А. Храпак, В. И. Молотков, О.Ф. Петров, "Пылевая плазма", УФН, 174:5 (2004), 495-544.

[69] Д. Н. Зубарев, В. Г. Морозов, И. П. Омелян, М. В. Токарчук, "О кинетических уравнениях для плотных газов и жидкостей”, ТМФ, 87:1 (1991), 113-129.

[70] Д. Н. Зубарев, В. Г. Морозов, И. П. Омелян, М. В. Токарчук, "Объединение кинетического и гидродинамического подходов в теории плотных газов и жидкостей”, ТМФ, 96:3 (1993), 325-350.

[71] M. V. Tokarchuk, I. P. Omelyan, A. E. Kobryn, "A consistent description of kinetics and hydrodynamics of systems of interacting particles by means of the nonequilibrium statistical operator method", Condens. Matter Phys., 1:4(16) (1998), 687-751.

[72] A. E. Kobryn, I. P. Omelyan, M. V. Tokarchuk, "The modified group expansions for construction of solutions to the BBGKY hierarchy", J. Statist. Phys., 92:5-6 (1998), 973-994.

[73] B. Markiv, I. Omelyan, M. Tokarchuk, "Consistent description of kinetics and hydrodynamics of weakly nonequilibrium processes in simple liquids", J. Statist. Phys., 155:5 (2014), $843-866$.

[74] Д. Н. Зубарев, В. Г. Морозов, "Формулировка граничных условий к цепочке Боголюбова с учетом локальных законов сохранения", ТМФ, 60:2 (1984), 270-279.

[75] V. G. Morozov, A. E. Kobryn, M. V. Tokarchuk, "Modified kinetic theory with consideration for slow hydrodynamical processes", Condens. Matter Phys., 1994, № 4, 117-127. 
[76] P. A. Hlushak, M. V. Tokarchuk, "Chain of kinetic equations for the distribution functions of particles in simple liquid taking into account nonlinear hydrodynamic fluctuations", Phys. A, 443 (2016), 231-245.

[77] I. R. Yukhnovskii, P. A. Hlushak, M. V. Tokarchuk, "BBGKY chain of kinetic equations, non-equilibrium statistical operator method and collective variable method in the statistical theory of non-equilibrium liquids", Condens. Matter Phys., 19:4 (2016), 43705, 18 pp.

[78] Д. Н. Зубарев, "Статистическая термодинамика процессов турбулентного переноса", TMФ, 53:1 (1982), 93-107.

[79] B. B. Markiv, R. M. Tokarchuk, P. P. Kostrobij, M. V. Tokarchuk, "Nonequilibrium statistical operator method in Renyi statistics", Phys. A, 390:5 (2011), 785-791.

[80] P. Kostrobij, R. Tokarchuk, M. Tokarchuk, B. Markiv, "Zubarev nonequilibrium statistical operator method in Renyi statistics. Reaction-diffusion processes", Condens. Matter Phys., 17:3 (2014), 33005, 9 pp.

[81] П. П. Костробий, А. В. Визнович, Б. Б. Маркив, М. В. Токарчук, “Обобщенные кинетические уравнения для плотных газов и жидкостей в методе неравновесного статистического оператора Зубарева и статистике Реньи", ТМФ, 184:1 (2015), 145-159.

[82] A. Rényi, Probability Theory, North-Holland Series in Applied Mathematics and Mechanics, 10, North-Holland, Amsterdam, 1970.

[83] Ю. Л. Климонтович, Введение в физику открытых систем, Янус-К, М., 2002.

[84] А. Г. Башкиров, "Энтропия Реньи как статистическая энтропия для сложных систем", TMФ, 149:2 (2006), 299-317.

[85] R. Luzzi, A. R. Vasconcellos, J. G. Ramos, "Non-equilibrium statistical mechanics of complex systems: an overview", Riv. Nuovo Cimento, 30:3 (2007), 95-158.

[86] A. R. Vasconcellos, J. G. Ramos, A. Gorenstein, M. U. Kleinke, T. G. Souza Cruz, R. Luzzi, "Statistical approach to non-Fickian diffusion", Internat. J. Modern Phys. B, 20:28 (2006), 4821-4841.

[87] Р. Р. Нигматуллин, “Дробный интеграл и его физическая интерпретация”, ТМФ, 90:3 (1992), 354-368.

[88] C. Tsallis, "Possible generalization of Boltzmann-Gibbs statistics", J. Statist. Phys., 52:1-2 (1988), 479-487.

[89] C. Tsallis (ed.), Introduction to Nonextensive Statistical Mechanics. Approaching a Complex World, Springer, New York, 2009.

[90] P. Turán (ed.), Selected Papers by Alfréd Rényi, v. 1, 2, Akadémiai Kiadó, Budapest, 1976.

[91] B. D. Sharma, D. P. Mittal, "New nonadditive measures of entropy for discrete probability distributions", J. Math. Sci., 10 (1975), 28-40.

[92] E. Aktürk, G. B. Bağci, R. Sever, Is Sharma-Mittal entropy really a step beyond Tsallis and Rényi entropies?, arXiv: cond-mat/0703277.

[93] C. Beck, "Superstatistics: theory and applications", Contin. Mech. Thermodyn., 16:3 (2004), 293-304.

[94] C. Beck, "Superstatistics: theoretical concepts and physical applications", Anomalous Transport: Foundations and Applications, eds. R. Klages, G. Radons, I. M. Sokolov, Wiley-VCH, New York, 2008, 433-457.

[95] В. В. Учайкин, Метод дробных производных, Артишок, Ульяновск, 2008.

[96] D. Korošak, B. Cvikl, J. Kramer, R. Jecl, A. Prapotnik, "Fractional calculus applied to the analysis of spectral electrical conductivity of clay-water system", J. Contain. Hydrol., 92:1-2 (2007), 1-9.

[97] R. Metzler, J. Klafter, "The random walk's guide to anomalous diffusion: a fractional dynamics approach", Phys. Rep., 339:1 (2000), 1-77.

[98] J. Bisquert, A. Compte, "Theory of the electrochemical impedance of anomalous diffusion", J. Electroanal. Chem., 499:1 (2001), 112-120. 
[99] T. Kosztołowicz, K. D. Lewandowska, "Hyperbolic subdiffusive impedance", J. Phys. A: Math. Theor., 42:5 (2009), 055004, 14 pp.

[100] J.-P. Bouchaud, A. Georges, "Anomalous diffusion in disordered media: statistical mechanisms, models and physical applications", Phys. Rep., 195:4-5 (1990), 127-293.

[101] R. R. Nigmatullin, "To the theoretical explanation of the 'Universal response", Phys. Stat. Sol. (b), 123:2 (1984), 739-745.

[102] R. R. Nigmatullin, "On the theory of relaxation for systems with 'remnant' memory", Phys. Stat. Sol. (b), 124:1 (1984), 389-339.

[103] R. R. Nigmatullin, "The realization of the generalized transfer equation in a medium with fractal geometry", Phys. Stat. Sol. (b), 133:1 (1986), 425-430.

[104] А. А. Хамзин, Р. Р. Нигматуллин, И. И. Попов, "Микроскопическая модель недебаевской диэлектрической релаксации. Закон Коула-Коула и его обобщение", ТМФ, 173:2 (2012), 314-332.

[105] R. Balescu, "Anomalous transport in turbulent plasmas and continuous time random walks", Phys. Rev. E, 51:5 (1995), 4807-4822.

[106] M. Tribeche, P. K. Shukla, "Charging of a dust particle in a plasma with a non extensive electron distribution function", Phys. Plasmas, 18:10 (2011), 103702.

[107] V.E. Tarasov, "Electromagnetic field of fractal distribution of charged particles", Phys. Plasmas, 12:8 (2005), 082106, 9 pp.

[108] А.С. Монин, "Уравнения турбулентной диффузии", Докл. АН СССР. Сер. геогр. и геофиз., 105 (1955), 256.

[109] G. M. Zaslavsky, "Chaos, fractional kinetics, and anomalous transport", Phys. Rep., 371:6 (2002), 461-580.

[110] С. Г. Самко, А. А. Килбас, О. И. Маричев, Интегралы и производные дробного порядка и некоторые их применения, Наука и техника, Минск, 1987.

[111] I. Podlubny, Fractional Differential Equations: An Introduction to Fractional Derivatives, Fractional Differential Equations, to Methods of Their Solution and Some of Their Applications, Mathematics in Science and Engineering, 198, Academic Press, San Diego, CA, 1999.

[112] V.E. Tarasov, "Fractional generalization of Liouville equations", Chaos, 14:1 (2004), $123-127$.

[113] V.E. Tarasov, "Fractional Liouville and BBGKI equations", J. Phys.: Conf. Ser., 7:7 (2005), 117-133.

[114] V.E. Tarasov, "Fractional systems and fractional Bogoliubov hierarchy equations", Phys. Rev. E, 71:1 (2005), 011102, 12 pp.

[115] V.E. Tarasov, "Fractional statistical mechanics", Chaos, 16:3 (2006), 033108, 7 pp.

[116] V.E. Tarasov, "Transport equations from Liouville equations for fractional systems", Internat. J. Modern Phys. B, 20:3 (2006), 341-353.

[117] K. Cottrill-Shepherd, M. Naber, "Fractional differential forms", J. Math. Phys., 42:5 (2001), 2203-2212.

[118] F. Mainardi, "Fractional calculus. Some basic problems in continuum and statistical mechanics", Fractals and Fractional Calculus in Continuum Mechanics (Udine, September 23-27, 1996), CISM International Centre for Mechanical Sciences. Courses and Lectures, 378, eds. A. Carpinteri, F. Mainardi, Springer, Vienna, 1997, 291-348.

[119] M. Caputo, F. Mainardi, "A new dissipation model based on memory mechanism", Pure Appl. Geophys., 91:1 (1971), 134-147.

[120] P. Kostrobij, B. Markovych, O. Viznovych, M. Tokarchuk, "Generalized diffusion equation with fractional derivatives within Renyi statistics", J. Math. Phys., 57:9 (2016), 093301, 8 pp.

[121] A. Compte, R. Metzler, "The generalized Cattaneo equation for the description of anomalous transport processes", J. Phys. A: Math. Gen., 30:21 (1997), 7277-7289. 
[122] I. I. Grygorchak, F O. Ivashchyshyn, M. V. Tokarchuk, N. T. Pokladok, O. V. Viznovych, "Modification of properties of $\mathrm{GaSe}\left(\beta\right.$-cyclodexterin $\left.\left(\mathrm{FeSO}_{4}\right)\right)$ Clathrat by synthesis in superposed electric and light-wave fields", J. Appl. Phys., 121:18 (2017), 185501, 7 pp.

[123] T.D. Lee, "The strongly interacting quark-gluon plasma and future physics", Nucl. Phys. A, 750:1 (2005), 1-8.

[124] M. Gyulassy, I. McLerran, "New forms of QCD matter discovered at RHIC", Nucl. Phys. A, 750:1 (2005), 30-63.

[125] E.V. Shuryak, "What RHIC experiments and theory tell us about properties of quark-gluon plasma?", Nucl. Phys. A, 750:1 (2005), 64-83.

[126] T. Hirano, N. Van Der Kolk, A. Bilandzic, "Hydrodynamics and flow", The Physics of the Quark-Gluon Plasma. Introductory Lectures, Lecture Notes in Physics, 785, eds. S. Sarkar, H. Satz, B. Sinha, Springer, New York, 2010, 139-178.

[127] T.S. Biró, E. Molnár, "Non-extensive statistics, relativistic kinetic theory and fluid dynamics", Eur. Phys. J. A, 48:11 (2012), 172, 11 pp.

[128] T. Osada, G. Wilk, "Nonextensive/dissipative correspondence in relativistic hydrodynamics", Progr. Theor. Phys. Suppl., 174 (2008), 168-172.

[129] T. Osada, G. Wilk, "Nonextensive hydrodynamics for relativistic heavy-ion collisions", Phys. Rev. C., 77:4 (2008), 044903, 20 pp.

[130] T. Osada, G. Wilk, "Nonextensive perfect hydrodynamics - a model of dissipative relativistic hydrodynamics?", Cent. Eur. J. Phys., 7:3 (2009), 432-443.

[131] T. Osada, "Relativistic hydrodynamical model in the presence of long-range correlations", Phys. Rev. C., 81:2 (2010), 024907, 8 pp.

[132] A. Lavagno, "Relativistic nonextensive thermodynamics", Phys. Lett. A, 301:1-2 (2002), $13-18$.

[133] G. Gianpiero, A. Lavagno, D. Pigato, "Nonextensive statistical effects in the quark-gluon plasma formation at relativistic heavy-ion collisions energies", Open Phys., 10:3 (2012), 594-601.

[134] A. Lavagno, D. Pigato, "Nonextensive statistical effects and strangeness production in hot and dense nuclear matter", J. Phys. G: Nucl. Part., 39:12 (2012), 125106, 16 pp.

[135] A. Lavagno, D. Pigato, "Nonextensive nuclear liquid-gas phase transition", Phys. A, 392:20 (2013), 5164-5171.

[136] Х. Умедзава, Х. Мацумото, М. Татики, Термополевая динамика и конденсированные состояния, Мир, М., 1985.

[137] L. Leplae, H. Umezawa, F. Mancini, "Derivation and application of the boson method in superconductivity", Phys. Rep., 10:4 (1974), 151-272.

[138] Y. Takahashi, H. Umezawa, "Thermo field dynamics", Collect. Phenom., 2:2 (1975), 55-80; reprinted in Internat. J. Modern Phys. B, 10:13-14 (1996), 1755-1805.

[139] T. Arimitsu, H. Umezawa, "A general formulation of nonequlibrium thermo field dynamics", Progr. Theor. Phys., 74:2 (1985), 429-432.

[140] T. Arimitsu, H. Umezawa, "Non-equilibrium thermo field dynamics", Progr. Theor. Phys., 77:1 (1987), 32-52.

[141] T. Arimitsu, "A canonical formalism of dissipative quantum systems. Non-equilibrium thermofield dynamics", Condens. Matter Phys., 4 (1994), 26-88.

Поступила в редакцию 25.04.2017, после доработки 19.05.2017 